Pacific Journal of Mathematic 


\section{SPLITTING HOMOTOPY IDEMPOTENTS WHICH HAVE ESSENTIAL FIXED POINTS}

\section{Ross GeoghegaN}

We consider the problem of whether every homotopy idempotent $f: X \rightarrow X, X$ being a finite CW complex, splits as a domination by $X$ of some $\mathrm{CW}$ complex $Y$. This problem has a history (which we explain) both in abstract homotopy theory and in geometric topology. If $f$ is a pointed homotopy idempotent, it is known that $f$ splits; if $X$ is permitted to be infinite-dimensional, it is known that $f$ need not split; and the obstruction to splitting is describable entirely in $f_{\sharp}: \pi_{1}(X, x) \rightarrow \pi_{1}(X, x)$. The difficulty, then, lies in requiring $X$ to be finite and permitting $f$ to be merely freely homotopic to $f^{2}$.

Our idea is to compare the fixed point theory of $f$ (this is where we use the fact that $X$ is finite) with its homotopy theory. We apply a theorem about the fundamental-group behavior of a homotopy idempotent which has essential fixed points, which we proved in the preceding paper. We believe that this theorem may eventually be used to prove our conjecture that $f$ splits when the Lefschetz number $L(f)$ is nonzero. In the present paper we only succeed in getting part of the way to such a result, by showing (Theorems 1.16 and 1.17) just how subtle a counter-example to the conjecture would have to be.

The problem of whether every homotopy idempotent on a finite complex splits is equivalent to the well-known problem in shape theory of whether every FANR is a pointed FANR (equivalently: does a compactum shape dominated by a complex have the shape of a complex?) In those terms, we are looking at the case of FANR's whose Cech Euler characteristic is nonzero.

1. Background and results. This paper is motivated by the following

Problem 1.1. Does every homotopy idempotent on a finite $\mathrm{CW}$ complex split? ${ }^{1}$

Before explaining the problem's significance, and our contribution to its solution, we define terms. $f: X \rightarrow X$ is a homotopy idempotent if $f$ is homotopic to $(\simeq) f^{2}(\equiv f \circ f)$. Whenever $X$ dominates a space $Y$ we find homotopy idempotents on $X$ : if $X \underset{d}{\stackrel{u}{\leftrightarrows}} Y$ are maps such that $d \circ u \simeq 1_{Y}$, then $u \circ d$ is clearly a homotopy 
idempotent. Now assume $X$ has the homotopy type of a CW complex; then the homotopy idempotent $f$ on $X$ splits if there is a space $Y$ and maps $u$ and $d$ as above such that $u \circ d \simeq f$. By a well-known theorem of Whitehead [13] such a space $Y$ must also have the homotopy type of a CW complex. Problem 1.1 deals with the case in which $X$ is (or has the homotopy type of) a finite CW complex. Note that it is not assumed that the maps and homotopies mentioned above preserve any base point: it will become clear that this is at the core of the problem.

Problem 1.1 seems to be of interest in two quite separate parts of topology. On the one hand, it is a problem in homotopy theory which has connections with combinatorial group theory. On the other, it is equivalent to a well-known geometrical problem about the shapes of compacta. Our investigation will use the NielsenReidemeister-Wecken theory of fixed points [1] as a tool. So, to organize the background, we begin with some exposition.

(A) The homotopy theory behind the problem:

We first drop the assumption that $X$ is finite, and discuss

Question 1.2. Does every homotopy idempotent on a CW complex split?

Here is a review of what is known. Let $X$ be a CW complex and $f$ a homotopy idempotent on $X$; assume $X$ is connected and (altering $f$ by a homotopy) that $f(x)=x$ for some $x \in X$ which we use as base point. By hypothesis there is a homotopy $H: f \underset{\omega}{\widetilde{\omega}} f^{2}$ where $\omega(t) \equiv H(x, t)$ is the loop at $x$ traced out by $x$.

Proposition 1.3. $f$ splits if and only if, in $\pi_{1}(X, x)$, we have $f_{\sharp}([\omega])=f_{\sharp}^{2}([\omega])$.

We sketch a proof of Proposition 1.3 in $\S 3$ but readers familiar with the problem will recognize that 1.3 is no more than a variation (useful here) on previously known results. Immediately we get

COROLLARY 1.4. $f$ splits if (i) $\omega$ is homotopically trivial, or (ii) $\pi_{1}(X, x)$ is abelian, or (iii) $f_{\sharp}([\omega]) \in$ image $\left(f_{\sharp}^{r}\right)$ for some $r \geqq 2$, or (iv) $\pi_{1}(X, x)$ is finite.

Proof. (i) is clear. $f_{\sharp}^{2}=T_{[\omega]} \circ f_{\#}$ (where $T_{g}(h) \equiv g^{-1} h g$ ) so (ii) is clear. If $f_{\sharp}([\omega])=f_{\sharp}^{r}([\sigma])$, then $f_{\sharp}^{2}([\omega])=f_{\sharp} \circ f_{\sharp}^{2} \circ f_{\sharp}^{r-2}([\sigma])=f_{\sharp}\left([\omega]^{-1}\right) . f_{\sharp}^{r}$ $([\sigma]) . f_{\sharp}([\omega])=f_{\sharp}([\omega])$, so (iii) is proved. (iv) is contained in (iii), since finiteness implies that $T_{[\omega]}$ takes image $\left(f_{\sharp}\right)$ isomorphically onto itself. 
Those are positive results, but there exist unsplittable homotopy idempotents. The simplest example is due independently to FreydHeller [6] and Dydak-Minc [2]. Before describing it we introduce the notation of HNN extensions. If $M$ is a group and $\alpha: M \rightarrow M$ is a monomorphism, the HNN extension $M_{\alpha}^{\circ}$ is obtained as follows: letting $\langle F ; R\rangle$ be a presentation for $M$, a presentation for $M_{\alpha}^{p}$ is $\left\langle F^{\prime} ; R^{\prime}\right\rangle$ where $F^{\prime}=F \cup\{t\}, t$ being a "new" generator, and $R^{\prime}=$ $R \cup\left\{t^{-1} m t \alpha\left(m^{-1}\right) \mid m \in M\right\} . \quad t$ is called the stable letter. (This is not the most general kind of HNN extension.) HNN extensions are discussed in [10], where the following is proved:

Proposition 1.5. If representatives are chosen from the right cosets in $M / \alpha(M), 1$ being chosen from the coset containing 1 , then every element of $M_{\alpha}^{\text {s }}$ can be written uniquely in the "normal form" $m_{0} t m_{1} t m_{2} t \cdots t m_{n} t^{-k}$ where $m_{0} \in M$ is arbitrary, while $m_{1}, \cdots, m_{n}$ are coset representatives, $k$ and $n$ being integers $\geqq 0$.

Now the example: we give details because we will need them.

EXAMPLE 1.6. Let $G$ be the group ${ }^{1}$ with generators $g_{\imath}, i=$ $0,1,2, \cdots$ and relations $g_{i}^{-1} g_{j} g_{i}=g_{j+1}$ whenever $i<j$; and let $\phi: G \rightarrow G$ take each $g_{i}$ to $g_{i+1}$. Clearly $\phi$ is a well-defined monomorphism, and $\dot{\phi}^{2}=T_{g_{0}} \circ \dot{\phi}$. Let $X_{0}=K(G, 1)$ and $f_{0}: X_{0} \rightarrow X_{0}$ be induced by $\dot{\phi}$. Then $f_{0} \underset{\omega_{0}}{\simeq} f_{0}^{2}$ where $\left[\omega_{0}\right]=g_{0}$. The natural homomorphism image $\left(\dot{\phi}^{2}\right)_{\rho \mid}^{p} \rightarrow$ image $(\phi)$ induced by inclusion on image $\left(\dot{\phi}^{2}\right)$ and taking the stable letter to $\phi\left(g_{0}\right)$ is clearly an isomorphism. So $\phi\left(g_{0}\right) \notin$ image $\left(\phi^{2}\right)$, by 1.5 . So $f_{0}$ does not split, by 1.3 .

We remark that $G$ has a two generator/two relation presentation (see [4 page 83]).

Freyd and Heller observed ([3] or [6]) that this example is "contained in" all examples:

Proposition 1.7. Define $\beta: G \rightarrow \pi_{1}(X, x)$ by $\beta\left(g_{r}\right)=f_{\#}^{r}([\omega])$. Then $\beta \circ \dot{\phi}=f_{\sharp \circ \beta} . \quad \beta$ is a monomorphism if and only if $f$ fails to split.

The "only if" half of this is immediate from 1.3. The "if" half is proved in [3]: the reader without access to [3] can easily put together a proof using the material in $\S 2$ below. The relationship between Question 1.2 and combinatorial group theory is clearer in the following variation on Proposition 1.7.

Proposition 1.8. $f$ fails to split if and only if the natural

${ }^{1}$ Besides [2] and [6], this group $G$ occurs in an earlier unpublished example of R. J. Thompson, who constructs an infinite, finitely presented simple group $H$ containing $G$ as a subgroup. I thank R. Strebel for this information. 
homomorphism $\gamma$ : image $\left(f_{\sharp}^{2}\right)_{f \neq 1}^{\rho_{1}} \rightarrow \pi_{1}(X, x)$ induced by inclusion on image $\left(f_{\sharp}^{2}\right)$ and taking the stable letter to $f_{\sharp}[\omega]$ is a monomorphism, whose image is the subgroup of $\pi_{1}(X, x)$ generated by $f_{\sharp}([\omega])$ and image $\left(f_{\sharp}^{2}\right)$.

Note that the image is as described whether or not $f$ splits. Monomorphism is the point. Proposition 1.8 is proved in $\S 2$.

(B) Additional information in the finite-dimensional case:

In between Problem 1.1 (unsolved) and Question 1.2 (solved) lies the unsolved

Problem 1.9. Does every homotopy idempotent on a finitedimensional $\mathrm{CW}$ complex split? ${ }^{2}$

On this there are two significant results:

Proposition 1.10 ([6]). A homotopy idempotent on a finitedimensional $K(\pi, 1)$ splits.

This is a consequence of 1.7 together with the easily checked fact that the elements $g_{2 i+1} g_{2 i}^{-1}$ of $G$ generate an infinite-dimensional free abelian group. See [3] for details.

Proposition 1.11 ([3]). If $X$ is n-dimensional and $\tilde{X}$ is $(n-1)$ connected then $f$ splits. In particular a homotopy idempotent on a two-dimensional CW complex splits.

(C) Geometrical meaning of Problem 1.1:

We do not know of any geometrical reason for studying Problem 1.9, but Problem 1.1, the case of a finite complex, has geometic content.

Let the compact metric space $Z$ be shape dominated by the finite CW complex $X$ (see [4] for information on shape theory). Then there are shape morphisms $X \underset{u}{\stackrel{d}{\rightleftarrows}} Z$ such that $d \circ u$ is the identity shape morphism. The shape morphism $u \circ d$ is clearly idempotent, and, since $X$ is an ANR, is representable by a map $f: X \rightarrow X$ which must be a homotopy idempotent. If $f$ splits as $X \underset{u^{\prime}}{\stackrel{d^{\prime}}{\rightleftarrows}} Y$, with $Y$ a CW complex, then $d \circ u^{\prime}$ and $d^{\prime} \circ u$ are mutually inverse shape morphisms, so $Z$ is shape equivalent to $\mathrm{CW}$ complex. Conversely if $Z$ is shape equivalent to a $\mathrm{CW}$ complex $Y$ it is an easy exercise to show that $f$ splits through $Y$. Compact metric spaces shape

\footnotetext{
${ }^{2}$ Added in proof: Hastings and Heller have answered Problem 1.9 affirmatively.
} 
dominated by finite CW complexes are called FANR's (or ANSR's in [4]). The above says that the FANR $Z$ has the shape of a CW complexe if and only if any one of the homotopy idempotents on finite complexes associated with $Z$ splits. Similar considerations show that if there is an unsplittable homotopy idempotent $f$ on a finite complex $X$, and if $Z=\lim \{X \stackrel{f}{\leftarrow} X \stackrel{f}{\leftarrow} \cdots\}$ then $Z$ does not have the shape of a $\mathrm{CW}$ complex.

Now a compact metric space $Z$ can be embedded in $\boldsymbol{R}^{n}$ or $Q$ (the Hilbert Cube) so as to have an $I$-regular neighborhood [11], [12] if and only if $Z$ is shape equivalent to a $\mathrm{CW}$ complex. Thus we have

Proposition 1.12. The following are equivalent: (a) every homotopy idempotent on a finite CW complex splits; (b) every FANR can be embedded in $\boldsymbol{R}^{n}$ or $Q$ so as to have an I-regular neighborhood.

For sharper information on the kind of embeddings see [11] and [12].

Another related matter is the following. If the pointed connected compact metric space $(Z, z)$ is pointed shape dominated by a pointed finite CW complex, $(Z, z)$ is called a pointed FANR. It is a fundamental problem in shape theory to decide whether $Z$ an FANR and $z \in Z$ imply $(Z, z)$ a pointed FANR. In [9] we explain the following:

Proposition 1.13. The following are equivalent: (a) every homotopy idempotent on a finite $\mathrm{CW}$ complex splits; (b) every FANR is a pointed FANR, with respect to any base point.

(D) The fixed point theory behind the problem:

In the preceding paper [7] we studied the fixed point theory of a homotopy idempotent on a finite complex $X$. The reader unfamiliar with fixed point theory as expounded, for example, in [1], should turn to $\S 2$ of [7] for a review. Here we merely remark: that certain fixed points of a map $f: X \rightarrow X$ are called "essential"; that if the Lefschetz number, $L(f)$, is nonzero then $f$ has at least one essential fixed point; and that for compact PL manifolds $X$ of dimension $\geqq 3$, $f$ has an essential fixed point if and only if $f$ is not homotopic to a fixed-point-free map.

We continue to consider a finite connected $\mathrm{CW}$ complex $X$, and $f: X \rightarrow X$ such that $f \simeq f^{2}$. From [7; Theorem 1.2] we quote:

Proposition 1.14. Let $f$ have an essential fixed point $x$ and, 
using $x$ as based point, let $H: f \underset{\omega}{\simeq} f^{2}$. Then there are integers $0<m<n$ and a loop $\sigma$ based at $x$ such that, in $\pi_{1}(X, x),[\omega]^{m}=[\sigma]$. $f_{\sharp}^{n}[\sigma]^{-1}$.

Here is an instructive example. Let $X$ be $S^{1} \vee S^{1}$, and let $f$ be the map which sends $\alpha$ to $\alpha$ and $\beta$ to $\alpha^{-1} \beta \alpha$ ( $\alpha$ and $\beta$ being the two "loops"). Then $f \simeq 1_{x}$ so $f$ is a splittable homotopy idempotent. Moreover $L(f) \neq 0$, so some fixed point of $f$ is essential. The wedge point $x$ is not essential; if it were, then, applying $f_{\#}$ to the equation in Proposition 1.14 and abelianizing, we would have $m\{\alpha\}=0$, which is false in $\left[\pi_{1}(X, x)\right]_{a b} \cong \boldsymbol{Z} \oplus \boldsymbol{Z}$. However the fixed point $\boldsymbol{y}$ half-way around $\beta$ is essential; with respect to $y f$ is pointed homotopic to $f^{2}$. This example is also useful in understanding 1.3: if $x$ is base point $\omega=\alpha$; if $y$ is base point $\omega=$ the constant loop; either way $f_{\sharp}([\omega])=f_{\sharp}^{2}([\omega])$.

We will apply Proposition 1.14 via

CoROLlaRy 1.15. With notation as in 1.14, let $K$ be the subgroup of $\pi_{1}(X, x)$ generated by $f_{\sharp}([\omega])$ and image $\left(f_{\sharp}^{2}\right)$. Then $f_{\sharp}^{2}([\omega])^{m}$ is a commutator in $K$ for some $m>0$.

Proof. By 1.14, $f_{\sharp}^{2}\left([\omega]^{m}\right)=f_{\sharp}^{2}([\sigma]) f_{\sharp}\left([\omega]^{-n}\right) f_{\sharp}^{2}\left([\sigma]^{-1}\right) f_{\sharp}\left([\omega]^{n}\right)$.

One consequence is to rule out the "universal example" of 1.6 and 1.7 in the following sense.

TheOREM 1.16. Let $f: X \rightarrow X$ be a homotopy idempotent on a finite complex, and let $f$ have an essential fixed point $x$. Then $f_{\sharp}: \pi_{1}(X, x) \rightarrow \pi_{1}(X, x)$ is not conjugate to the homomorphism $\phi: G \rightarrow G$ described in Example 1.6.

Theorem 1.16 is proved in $\S 2$.

Another consequence comes from combining 1.8 and 1.15:

THEOREM 1.17. Let $f: X \rightarrow X$ be a homotopy idempotent on a finite complex, and let $f$ have an essential fixed point $x$. Let $f \underset{\omega}{\widetilde{\omega}} f^{2}$, let $K$ be the subgroup of $\pi_{1}(X, x)$ generated by $f_{\sharp}([\omega])$ and image $\left(f_{\sharp}^{2}\right)$, and let $p: K \rightarrow K_{a b}$ be the natural projection. Suppose $f$ fails to split. Then $p\left(f_{\sharp}([\omega])\right)$ has infinite order in $K_{a b}$ while $p\left(f_{\sharp}^{2}[[\omega])\right)$ has finite order in $K_{a b}$.

Proof. The finite order comes from 1.15. The infinite order comes from 1.8, using the fact that when $M_{\alpha}^{p}$ is abelianized, the 
stable letter goes to a generator of a $Z$-summand.

We conjecture that the situation described in Theorem 1.17 cannot happen, and hence that $f$ (with an essential fixed point) must split. A proof of this conjecture has eluded us so far.

2. Proofs of Proposition 1.8 and Theorem 1.16. To simplify notation in Proposition 1.8, write $\pi=\pi_{1}(X, x), F=f_{\#}$ and $z=[\omega]$. Then $\gamma$ : image $\left(F^{2}\right)_{F \mid}^{p} \rightarrow \pi$ is inclusion on image $\left(F^{2}\right)$, and takes the stable letter $t$ to $F(z)$.

Proof of Proposition 1.8. First “if". Suppose $f$ splits. Then, by $1.3, F(z)=F^{2}(z)$, so $\gamma(t) \in$ image $\left(F^{2}\right)$. But $\gamma$ is mono, and, by 1.5, $t \notin$ image $\left(F^{2}\right)$. Contradiction.

To prove "only if", we first note that if $f$ fails to split, then no power of $F(z)$ lies in image $\left(F^{2}\right)$. Because, suppose $F\left(z^{k}\right)=F^{2}(y)$. Then $F^{2}\left(z^{k}\right)=F\left(z^{-1} F(y) z\right)=F\left(z^{-1}\right) \cdot F^{2}(y) \cdot F(z)=F\left(z^{k}\right)$. So, by 1.3, the homotopy idempotent $f^{k}$ splits $\left(f^{k} \underset{\omega^{k}}{\widetilde{f}} f^{2 k}\right)$, hence $f$ splits since $f \simeq f^{n}$.

Every element of image $\left(F^{2}\right)_{F \mid}^{t}$ has normal form $\tau=m_{0} t m_{1} t m_{2} t \ldots$ $t m_{n} t^{-k}$ as in Proposition 1.5. Let $i(\tau)=n+k$. Suppose ker $\gamma \neq\{1\}$, and pick $\tau \in \operatorname{ker} \gamma$ so that $\tau \neq 1$ and $i(\tau)$ is minimal among all such.

Case 1. No $t$ 's occur in $\tau$. Then $\tau=m_{0}, \gamma(\tau)=1=\gamma\left(m_{0}\right)$ so $m_{0}=1=\tau$. Contradiction.

Case 2. Negative powers of $t$ occur in $\tau$, but no positive powers: then $\tau=m_{0} t^{-k}$ and $\gamma(\tau)=1$, so $F\left(z^{k}\right) \in$ image $\left(F^{2}\right)$ which we have seen to be impossible.

Case 3. Positive powers of $t$ occur in $\tau$, but no negative powers. Then $\tau=m_{0} t m_{1} t m_{2} \cdots t m_{n}$. For all $m \in$ image $\left(F^{2}\right), t^{-1} m t=F(m)$, so $m t=t F(m)$. Pulling the $m$ 's to the right we get $\tau=t^{n} m$ where $m \in$ image $\left(F^{2}\right) . \quad \gamma(\tau)=1=F\left(z^{n}\right) m$, so $F\left(z^{n}\right) \in$ image $\left(F^{2}\right)$ which, as has been said, cannot happen.

Case 4. Both positive and negative powers of $t$ occur in $\tau$. Then $\tau=m_{0} t m_{1} t m_{2} \cdots t m_{n} t^{-k}=t F\left(m_{0}\right) m_{1} t m_{2} \cdots t m_{n} t^{-k}$. So $t^{-1} \tau t=$ $F\left(m_{0}\right) m_{1} t m_{2} \cdots t m_{n} t^{-(k-1)}$ is also in $\operatorname{ker} \gamma$. Yet $i\left(t^{-1} \tau t\right)=i(\tau)-2$. Since $i(\tau)$ is minimal, $t^{-1} \tau t=1$. Hence $\tau=1$. Contradiction.

REMARK 2.1. The above proof can be adapted trivially to show that if $f$ fails to split then the subgroup of $\pi_{1}(X, x)$ generated by $[\omega]$ and image $\left(f_{\sharp}\right)$ is also an HNN extension. But this is not "if 
and only if": a constant map from the circle to itself is a splittable homotopy idempotent for which the same property holds (if $\omega$ is chosen to generate $\pi_{1}$ ).

We now turn to Theorem 1.16. Recall $\phi: G \rightarrow G$ in Example 1.6. The reader is invited to check the following (or see [3]):

LeMma 2.2. (a) Every $g \in G$ can be written

$$
g=g_{i_{1}}^{n_{1}} \cdots g_{i_{k}}^{n_{k}} 1 g_{i_{k+1}}^{-n_{k+1}} \cdots g_{i_{r}}^{-n_{r}}
$$

where each $n_{i}$ is a positive integer, $i_{1}<i_{2}<\cdots<i_{k}$, $i_{k+1}>i_{k+2}>$ $\cdots>i_{r}$,

(b) If $p: G \rightarrow G_{a b} \cong \boldsymbol{Z} \oplus \boldsymbol{Z}$ is projection, $p\left(g_{0}\right)=(1,0)$ and $p\left(g_{i}\right)=(0,1)$ for all $i>0$.

Proof of Theorem 1.16. Suppose $f_{\#}$ is conjugate to $\phi$. To

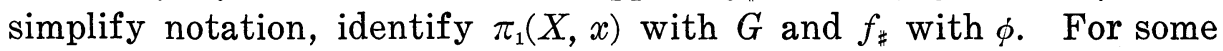
$\omega, f \underset{\omega}{\simeq} f^{2}$. Let $[\omega]$ be identified with $g \in G$. Then $\phi^{2}=T_{g_{0}} \circ \dot{\phi}=$ $T_{g} \circ \phi$, so $g_{0}^{-1} g$ commutes with $\phi(G)$. By Corollary $1.15, \phi^{2}(g)^{m}$ is a commutator in $\phi(G)$, hence $\phi(g)^{m}$ is a commutator in $G, \phi$ being mono. By Lemma 2.2(a) we can write $g=g_{i_{1}}^{n_{1}} \cdots g_{i_{k}}^{n_{k}} 1 g_{i_{k+1}}^{-n_{k+1}} \cdots g_{i_{r}}^{-n_{r}}$ where each $n_{i}$ is a positive integer, $i_{1}<\cdots<i_{k}, i_{k+1}>\cdots>i_{r}$. If $s>\max \left\{i_{1}, \cdots, i_{r}\right\}$ the relations in $G$ give

$$
\begin{aligned}
& g_{s}\left(g_{0} g^{-1}\right)=g_{0} g_{i_{r}}^{n_{r}} \cdots g_{i_{k+1}}^{n_{k+1}} g_{s+1+n_{r}+\cdots+n_{k+1}} 1 g_{i_{k}}^{-n_{k}} \cdots g_{i_{1}}^{-n_{1}} \\
& \left(g_{0} g^{-1}\right) g_{s}=g_{0} g_{i_{r}}^{n_{r}} \cdots g_{i_{k+1}}^{n_{k+1}} g_{s+n_{1}+\cdots+n_{k}} 1 g_{i_{k}}^{-n_{k}} \cdots g_{i_{1}}^{-n_{1}} .
\end{aligned}
$$

Hence $1+n_{k+1}+\cdots+n_{r}=n_{1}+\cdots+n_{k}$.

But, since $\phi(g)^{m}$ goes to $0 \in \boldsymbol{Z} \oplus \boldsymbol{Z}$ under abelianization, so does $\phi(g)$. Hence, by Lemma 2.2(b), $n_{1}+\cdots+n_{k}=n_{k+1}+\cdots+n_{r}$. This gives a contradiction.

3. Proof of Proposition 1.3. An inverse sequence of groups $G_{1} \stackrel{f_{1}}{\leftarrow} G_{2} \stackrel{f_{2}}{\leftarrow} \ldots$ is Mittag-Leffler (ML) if for each $m$, there exists $n$ such that image $\left(G_{m+r} \rightarrow G_{n}\right)$ is independent of the nonnegative integer $r$.

As in $\S 2$, let $\pi=\pi_{1}(X, x), F=f_{\sharp}$ and $z=[\omega]$. We are to prove that $f$ splits if and only if $F(z)=F^{2}(z)$.

LEMma 3.1. $F(z)=F^{2}(z)$ if and only if the sequence $\pi \stackrel{F}{\leftarrow} \pi \stackrel{F}{\leftarrow} \ldots$ is ML.

Proof. If the sequence is ML then for some $r \geqq 1$ image $\left(F^{r}\right)=$ image $\left(F^{r+1}\right)$. So $F^{r}(z)=F^{r+1}(y)$ for some $y$; hence $F(z)=F^{2}(y)$, since we may conjugate by $z^{r-1}$. So $F^{2}(z)=F\left(z^{-1} F(y) z\right)=$ 
$F(z)^{-1} F^{2}(y) F(z)=F(z)$.

Conversely, letting $T_{y}(\omega)=y^{-1} \omega y$, note that if $F(z)=F^{2}(z)$, then image $\left(F^{3}\right)=T_{\left.F^{(z)}\right)}\left(\right.$ image $\left.\left(F^{2}\right)\right)=T_{F^{2}(z)}\left(\right.$ image $\left.\left(F^{2}\right)\right)=$ image $\left(F^{2}\right)$, so the sequence $\pi \stackrel{F}{\leftarrow} \pi \stackrel{F}{\leftarrow} \ldots$ is $\mathrm{ML}$.

Proof of Proposition 1.3. If $f$ splits as $X \underset{u}{\stackrel{d}{\rightleftarrows}} Y$ then the sequence $\pi \stackrel{F}{\leftarrow} \pi \stackrel{F}{\leftarrow} \ldots$ is cofinal in the sequence $\pi_{1}(X, x) \stackrel{u_{\sharp}}{\longleftarrow} \pi_{1}(Y, y) \stackrel{d \sharp}{\longleftarrow}$ $\pi_{1}(X, x) \stackrel{u_{\sharp}}{\longleftarrow} \ldots$, as is $\pi_{1}(Y, y) \stackrel{(\text { dou })_{\sharp}}{\longleftarrow} \pi_{1}(Y, y) \stackrel{(\text { dou })_{\sharp}}{\longleftarrow} \ldots$. Since $d \circ u \simeq 1_{Y}$, the latter is $\mathrm{ML}$, hence so are the others.

Conversely, if the sequence $\pi \stackrel{F}{\leftarrow} \pi \stackrel{F}{\leftarrow} \ldots$ is ML it is well-known that $f$ splits. A proof can be found, for example, in [4] Theorem 9.2.2 $(5 \rightarrow 3)$ where only the case of finite $X$ is explicitly considered. But the proof for infinite $X$ is the same. For other proofs see [8] and [5].

\section{REFERENCES}

1. R. Brown, The Lefschetz Fixed Point Theorem, Scott Foresman, Glenview and London 1971.

2. J. Dydak, A simple proof that pointed connected FANR-spaces are regular fundamental retracts of ANR's, Bull. Acad. Polon. Sci., Ser. Math., Astronom., Phys., 25 (1977), 55-62.

3. J. Dydak and H. Hastings, Homotopy idempotents on two-dimensional complexes split, Proceedings of International Conference on Geometric Topology, Warsaw 1980 (to appear).

4. J. Dydak and J. Segal, Shape Theory, Lecture Notes in Mathematics, volume 688, Springer-Verlag, Berlin, 1978.

5. D. A. Edwards and R. Geoghegan, Stability theorems in shape and prohomotopy, Trans. Amer. Math. Soc., 222 (1976), 389-403.

6. P. Freyd and A. Heller, Splitting homotopy idempotents II, (preprint).

7. R. Geoghegan, The homomorphism on fundamental group induced by a homotopy idempotent having essential fixed points, Pacific J. Math., 92 (1981), to appear.

8. — Elementary proofs of stability theorems in shape and prohomotopy, Gen. Topo'ogy and Appl., 8 (1978), 265-281.

9. - The problem of pointed versus unpointed domination in shape theory, Topology Proceedings, 3 (1978), 95-107.

10. R. Lyndon and P. Schupp, Combinatorial Group Theory, Ergebnisse der Mathematik und ihrer Grenzgebiete, vol. 89, Springer-Verlag, Berlin and New York, 1977.

11. L. C. Siebenmann, Regular (or canonical) open neighborhoods, Gen. Topology and Appl., 3 (1973), 51-62.

12. L. C. Siebenmann, L. Guillou and H. Hähl, Les voisinages reguliers ouverts, Ann. Sci. E.N.S. (4) 6 (1973), 253-293.

13. J. H. C. Whitehead, A certain exact sequence, Ann. Math., 52 (1950), 51-110.

Received November 13, 1979. Supported in part by NSF Grant.

Institute FOR AdVANCEd StUdy

PRINCETON, NJ 08540

AND

State University of New York at Binghamton

Binghamton, NY 13901 



\section{PACIFIC JOURNAL OF MATHEMATICS}

\section{EDITORS}

DONALD BABBITT (Managing Editor)

University of Galifornia

Los Angeles, California 90024

Hugo RossI

University of Utah

Salt Lake City, UT 84112

C. C. MOORE AND ANDREW OGG

University of California

Berkeley, CA 94720
J. DugundJI

Department of Mathematics University of Southern California Los Angeles, California 90007

R. FinN and J. Milgram Stanford University Stanford, California 94305

\section{ASSOCIATE EDITORS}

R. ARENS

E. F. BeCKenbaCh

B. H. NEUManN

F. WOLF

K. YosHIDA

\section{SUPPORTING INSTITUTIONS}

UNIVERSITY OF ARIZONA

UNIVERSITY OF BRITISH COLUMBIA

CALIFORNIA INSTITUTE OF TECHNOLOGY

UNIVERSITY OF CALIFORNIA

MONTANA STATE UNIVERSITY

UNIVERSITY OF NEVADA, RENO

NEW MEXICO STATE UNIVERSITY

OREGON STATE UNIVERSITY
UNIVERSITY OF OREGON

UNIVERSITY OF SOUTHERN CALIFONIA

STANFORD UNIVERSITY

UNIVERSITY OF HAWAII

UNIVERSITY OF TOKYO

UNIVERSITY OF UTAH

WASHINGTON STATE UNIVERSITY

UNIVERSITY OF WASHINGTON 


\section{Pacific Journal of Mathematics}

\section{Vol. 95, No. $1 \quad$ September, 1981}

John Allen Beachy and William David Blair, On rings with bounded

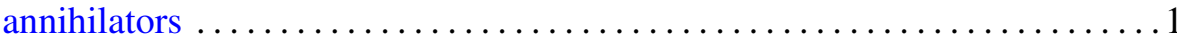

Douglas S. Bridges, A constructive look at positive linear functionals on

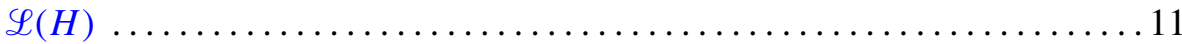

Muneo Chō and Makoto Takaguchi, Boundary points of joint numerical ranges

W. J. Cramer and William O. Ray, Solvability of nonlinear operator equations

Lester Eli Dubins and Gideon Schwarz, Equidiscontinuity of

Borsuk-Ulam functions

Maria Fragoulopoulou, Spaces of representations and enveloping 1.m.c.

*-algebras

Robert F. Geitz and J. Jerry Uhl, Jr., Vector-valued functions as families of scalar-valued functions

Ross Geoghegan, The homomorphism on fundamental group induced by a homotopy idempotent having essential fixed points

Ross Geoghegan, Splitting homotopy idempotents which have essential fixed points

Paul Jacob Koosis, Entire functions of exponential type as multipliers for

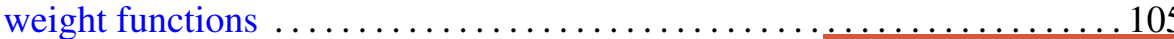

David London, Monotonicity of permanents of certain doubly stochastic matrices

Howard J. Marcum, Two results on cofibers

Giancarlo Mauceri, Zonal multipliers on the Heisenberg group

Edward Wilfred Odell, Jr. and Y. Sternfeld, A fixed point theorem in $c_{0} \quad \ldots 161$

Bernt Karsten Oksendal, Brownian motion and sets of harmonic measure zero

Andrew Douglas Pollington, The Hausdorff dimension of a set of normal numbers

Joe Repka, Base change lifting and Galois invariance ...

Gerald Suchan, Concerning the minimum of permanents on doubly stochastic circulants

Jun-ichi Tanaka, On isometries of Hardy spaces on compact abelian groups

Aaron R. Todd, Quasiregular, pseudocomplete, and Baire spaces 